\title{
Evolución de la cooperación europea en inteligencia
}

\section{The evolution of European cooperation in intelligence}

\author{
ANTONIO M. DÍAZ FERNÁNDEZ \\ Profesor Titular de Ciencia Política en la Universidad de Cádiz \\ Paseo Carlos III, 3, $2^{a}$ planta Edificio Constitución 1812 \\ (Antiguo "Cuartel de La Bomba") 11003, Cádiz, España \\ adiaz@ubu.es
}

RESUMEN La cooperación entre los países europeos en materia de inteligencia no se inicia tan recientemente como podría parecer. La difícil relación mantenida durante la guerra fría no podría hacernos pensar que los europeos colaborasen intensamente y se intercambiasen información e inteligencia de forma intensa. Este artículo describe la evolución de esta cooperación.

Palabras clave cooperación, inteligencia, Europa.

ABSTRACT European cooperation on intelligence among European countries does not start as late as it sounds. The difficult relationship maintained within Europe during the cold war may not suggest the existence of a strong collaboration and information and intelligence exchange. This article describes the evolution of this cooperation.

Keywords cooperation, intelligence, Europe.

La historia de la construcción europea tiene su origen remoto en el intento de que no se volviera a producir un conflicto entre europeos. Para eso se controlaron los elementos principales de la industria del armamento: el acero y el carbón. Mucho ha llovido desde entonces y la historia en común

* $\quad$ Artigo recebido em: 21/12/2011. Autor convidado. 
de los europeos es de una creciente protección mutua. Desde la firma de los tratados constituyentes en Roma, la mutua dependencia y la autorregulación cimentaron la base para que Europa viviese el período de paz más largo de su historia. Sin embargo, la seguridad por sí sola nunca fue un aspecto importante en la agenda comunitaria que quedaba colmada por políticas y acciones de eminente carácter político y económico. No obstante, el entramado comunitario sí, favoreció el desarrollo de algunas iniciativas, ya que no podemos olvidar que en la base de la creación del proceso de construcción europeo está el evitar los fratricidas combates de antaño.

En la década de los setenta aparecen una nueva serie de amenazas como el alcance que cobraban el tráfico de estupefacientes o los atentados terroristas en Europa Occidental y que mostraron las inadaptaciones estructurales de Interpol. Esto llevó a que en el Consejo Europeo de Roma, en diciembre de 1975 y junio de 1976, los ministros de Justicia e Interior decidieran crear un foro para combatir el terrorismo que sería conocido como el Grupo Trevi y cuyo acrónimo nace de Terrorism, Radicalism, Extrermism and International Violence. A través de sus reuniones regulares proporcionó a las fuerzas policiales europeas el marco necesario para poder comunicarse e intercambiar información sobre crímenes transfronterizos así como compartir mejores prácticas para afrontarlas.

Este intento de combinar servicios de inteligencia y clásicas agencias policiales contra el crimen internacional -que en alguna medida supuso Trevi- se estrechó y fortaleció gracias a las modificaciones legislativas producidas sobre los servicios de inteligencia de algunos países europeos, especialmente, el Reino Unido y Alemania. Estas modificaciones permitieron que los servicios de inteligencia, previamente limitados en su actividad al campo político y militar, se situasen a la vanguardia de las actividades europeas de lucha policial contra el crimen organizado. Incluso la Comisión Europea, que por muchos años había evitado tratar el tema de los servicios de inteligencia, se dio cuenta del potencial de la cooperación en inteligencia contra el crimen que afectaba a los intereses económicos de la Unión.

Aunque sin duda fue el más importante, Trevi no fue el único marco de cooperación a nivel europeo. Desde 1971 también nos podemos encontrar con el Club de Berna que operaba de manera informal, con reuniones que tenían lugar en diferentes localidades e implicando a diferentes organizaciones que albergaban las reuniones de forma alternativa. En 1977 se establece el Grupo Kilowatt, que comprendía a los miembros de la Comunidad Europea, Canadá, Noruega, Suecia, Suiza, Estados Unidos e Israel. ${ }^{1}$ En este grupo el intercambio de información no se basaba en la reciprocidad, puesto que cada servicio de inteligencia nacional ponía a disposición de los demás países toda la información sobre terrorismo con la que contaba.

1 Documento del Consejo. The new challenges facing European intelligence: reply to the annual report of the Council, doc.A/1775, de 4 de junio de 2002. 
Esto favoreció enormemente a los países más pequeños que recibieron mucha más información e inteligencia y de más calidad que la que podían poner a disposición de los demás; como comprobaremos más adelante, este debate sobre qué nivel de intercambio debe existir se reproduce con enorme intensidad tras el 11-S. Otros grupos que representan importantes precedentes de la cooperación que vivimos en la actualidad fueron el Grupo de Trabajo Policial en Materia de Terrorismo (GTPMT), el grupo de Salzburgo y el grupo del mar del Báltico que muestra la geometría variable de esta cooperación.

Pero el hito en las políticas de seguridad interior en estos años - hasta el punto de inflexión de los atentados de septiembre de 2001 - queda marcado por la firma del Acuerdo Schengen el 14 de junio de 1985 y que entró en vigor el 19 de junio de 1990. Bélgica, Holanda, Luxemburgo, Francia y Alemania acuerdan instaurar un régimen de libre circulación para todos los Estados signatarios y establecer facilidades en los controles fronterizos, una mayor coordinación en la lucha contra el tráfico de estupefacientes, crimen organizado y la inmigración ilegal. Cinco años más tarde se estableció en Acuerdo de Implementación Schengen que incluyó aspectos esenciales de esta cooperación y creó una base de datos común electrónica, denominada Sistema de Información Schengen (SIS). ${ }^{2}$

El Acta Única Europea firmada en 1986 constituyó otro destacado paso en esta cooperación europea y en la conciencia de la importancia del intercambio de información. Tras la firma del Acta se crearon grupos de trabajo como el ad hoc sobre inmigración, creado ese mismo año e integrado por los ministros responsables de esta materia, o el Comité Europeo de Lucha contra la Droga (CELAD), que quedó establecido en el Consejo Europeo de Estrasburgo en 1989 con el objetivo de formular la estrategia principal para la lucha contra el tráfico de estupefacientes. ${ }^{3}$ También nace el Grupo de Aduanas de Asistencia Mutua (GAM) encargado de cuestiones aduaneras $y$, finalmente, se configura un Consejo formado por los ministros de Justicia de los Estados miembros que celebra reuniones periódicas ocupándose de la cooperación judicial, penal y civil, junto con determinados aspectos de la cooperación política europea. ${ }^{4}$

La necesidad de garantizar la libertad de movimientos en el mercado europeo, desembocó en el establecimiento del Grupo de Coordinadores, creado en el Consejo Europeo de Rodas de 1988, que encargó al grupo intergubernamental de coordinación denominado "libre circulación de las personas" que propusiera medidas encaminadas a conjugar la libre circulación de las personas y la seguridad ciudadana, una vez eliminados los controles en las fronteras interiores de la Comunidad. Este grupo propuso

\footnotetext{
Comunicación de la Comisión al Parlamento Europeo y al Consejo. COM 720 Final, 2001.

Conclusiones de la Presidencia del Consejo Europeo de Estrasburgo de 1989.

Doc. CIRC. 3624/89
} 
un programa de trabajo cuyo resultado fue el conocido como Documento de Palma, adoptado por el Consejo Europeo de julio de 1989. En él se recomendaba un enfoque más coordinado en diversos aspectos de la cooperación en materia de Justicia e Interior y una serie de medidas que comportaban la correspondiente creación de sistemas de información para el control y el seguimiento de ciudadanos.

La Cumbre ministerial de Dublín de junio de 1990 aprobó, en base al programa de acción del Trevi, la creación de la Unidad de Inteligencia Europea contra el Tráfico de Drogas (EDU). Su misión consistía en la recopilación y análisis de información suministrada por los Estados miembros en lo relativo al tráfico de drogas a nivel europeo. Con el paso del tiempo, a esta unidad se le otorgó el nombre de Unidad de Europol contra las Drogas, al mismo tiempo que fue adquiriendo nuevas competencias en materias como el robo de vehículos, organizaciones clandestinas de inmigración y tráfico de sustancias radioactivas.

Se vislumbra entonces la creciente complejidad de la colaboración en el seno de la Comunidad Europea en diferentes niveles y en la que la información tenía un papel destacado. No obstante, distaba de ser una situación idílica ya que los diversos grupos de trabajo surgidos a lo largo del tiempo trabajaban por separado y elaboraban sus informes para ministros que integraban estructuras distintas dentro de la Comunidad sin alcanzar una visión centralizada.

\section{De Maastricht a Niza (1992-2000). La seguridad interior y exterior entran en la agenda europea}

En Maastricht nace una estructura polémica, pero que sirvió para avanzar en la construcción europea, conocida como pilares. Los pilares son agrupaciones de competencias en las que a partir del Tratado de Maastricht se dividen las áreas de trabajo de la Unión. El primero de ellos contiene las políticas comunitarias, el segundo se ocupa de las políticas intergubernamentales de exterior, seguridad y defensa y el tercero el que asume los asuntos de justicia e interior; sin embargo, ninguno de ellos supuso un desarrollo específico de las capacidades de inteligencia de la Unión.

En 1997 se firma el Tratado de Ámsterdam, modificando lo contenido en Maastricht un lustro antes. Su artículo 11 otorga plena carta de naturaleza a la Política Exterior y de Seguridad Común (PESC) al entregar a la Unión la definición de la política exterior y de seguridad común, para lo que establece la cooperación como un elemento axial. En Ámsterdam se precisa el contenido de las "Operaciones Petersberg" de la Unión Europea Occidental (UEO) instituidas en Maastricht en 1992 (humanitarias, de mantenimiento y restablecimiento de la paz y gestión de crisis) y para lo cual se establece por primera vez la necesidad de capacidades de inteligencia que eran mínimas 
y hasta entonces proporcionadas por la OTAN. Por esto, tras Kosovo, se produjo una reunión bilateral entre Francia y el Reino Unido, a finales de 1998, en la cual se expuso la necesidad de poner en marcha una Política de Defensa incluida en la PESC. En esta Declaración de Saint-Malo se establece la necesidad de "suministrar las estructuras y capacidades adecuadas para el análisis de las situaciones, fuentes de inteligencia y capacidades para planeamiento estratégico relevante sin una duplicación innecesaria". ${ }^{5}$

La llegada de estas capacidades de inteligencia no se hizo esperar. En diciembre de ese mismo año, en la cumbre de Helsinki, la Unión aprobó la creación de una Fuerza de Acción Rápida que incluiría capacidades de inteligencia, ${ }^{6}$ como la G-2, la división de inteligencia para recopilar información y producir asesoramientos y un acuerdo UE-OTAN en materia de inteligencia llamado BICES (Battlefield Information Collection and Exp/oitation System), ${ }^{7}$ que someramente, puede definirse como una red informática secreta de inteligencia en la que cada Estado aliado obtiene la información que necesita. En febrero de 2001 entra en vigor Niza e inmediatamente los tres nuevos cuerpos en la Secretaría del Consejo que representan un importante progreso para la Europa de la Defensa: el Comité Político y de Seguridad, el Comité Militar de la UE y el Estado Mayor de la UE y declarados operativos en el Consejo de Laeken en diciembre de 2001. En sendas decisiones de la UE y la OTAN en la cumbre de Washington y el Consejo Europeo de Colonia de 1999 ambas instituciones acuerdan desarrollar un sistema de cooperación basado en diferentes ámbitos, entre ellos el de un acuerdo sobre el intercambio de información y las modalidades de consulta y cooperación en períodos dentro y fuera de crisis, si bien sufrió algún problema por las medidas de seguridad para permitir el intercambio de información clasificada y cooperación en materia de seguridad. ${ }^{8}$

El consejo de la UEO de Marsella el 13 de diciembre de 2000 decidió ceder la organización operativa de gestión de crisis de la UEO a la UE, lo que completaría los instrumentos de la Unión en el marco de la PESD. Por estas modificaciones, del Tratado de Niza desaparecen de su artículo 17 toda referencia a la UEO y tanto el Centro de Satélites de Torrejón como el Instituto de Estudios de Seguridad de la UEO pasan desde el 1 de enero de 2002 a ser agencias de la Unión Europea. La UEO se integra así en el entramado de la Unión desarrollando un papel importante en incitar la cooperación en inteligencia entre los países europeos.

El tercer pilar también quedó afectado por Ámsterdam. Pero fue el Consejo de Cardiff de 15 y 16 de junio de 1998 quien invitó a Comisión y

5 Declaración conjunta sobre Defensa de la cumbre franco-británica en Saint-Malo, Francia, 4 de diciembre de 1998. http://www.fco.gov.uk/news/newstext.asp?1795

6 Véase Conclusiones de la Presidencia del Consejo Europeo de Helsinki de 1999

7 Véase "Linked Operations-Intelligence Centers Europe (LOCE)". http://www.fas.org/irp/program/disseminate/loce. $\mathrm{htm}$

8 Nota de Prensa de la OTAN n.022 de 14 de marzo de 2003 y Decisión del Consejo de la Unión Europea 2003/211/ CFSP, de 24 de febrero de 2003. 
Consejo a presentar en Viena en el mes de diciembre un "Plan de acción sobre la mejor manera de aplicar las disposiciones del Tratado de Ámsterdam relativas a la creación de un espacio de libertad, seguridad y justicia". ${ }^{9}$ Este Plan de Acción vio la luz en la capital austriaca y, entre otros elementos, contempla que se estudie la viabilidad de crear una base de datos sobre investigaciones pendientes, en el marco de las disposiciones de Europol, que permitiera evitar toda superposición entre las mismas y asociar varios servicios policiales europeos en la misma investigación, combinando así sus conocimientos y experiencia. ${ }^{10} \mathrm{El}$ Consejo Europeo era muy consciente de las carencias existentes en cooperación por lo que se estableció un Plan de Acción para darle un empuje sobre todo centrado en el combate del tráfico de drogas y el crimen organizado. Este Plan se aprueba durante el Consejo Especial de Justicia e Interior celebrado en Tampere, Finlandia, los días 15 y 16 de octubre 1999. ${ }^{11}$ Con el objetivo de reforzar el Plan de Acción de Viena, las conclusiones de la cumbre se plasmaban en diez retos para el progreso del establecimiento del área de Libertad, Seguridad y Justicia que se subdividen en 62 puntos de acción específicos que habría de ser revisado en 2004. ${ }^{12}$ Algunos eran prexistentes pero, sobre todo, Tampere ayuda a establecer nuevas vías de progreso para el área de Justicia e Interior. Supone así un importante hito de esta fase inicial de la cooperación europea ya que por primera vez los Estados miembros hacen constar que los ciudadanos europeos tienen derecho a esperar que la Unión afronte las diversas formas de delincuencia que representan una amenaza para su libertad y sus derechos civiles.

\section{Cooperación europea en inteligencia tras el 11-S}

La cooperación en materia de información e inteligencia se ha ido fraguando lentamente en el entramado comunitario y cómo un buen número de iniciativas ya estaban, si no adoptadas, al menos ya pergeñadas antes de los atentados del 11-S. La necesidad de incrementar esta cooperación ante la indiscutible amenaza del terrorismo internacional y el crimen organizado que se cernía sobre Europa era denunciada por los responsables políticos y de las agencias de seguridad desde hacía años. Sin embargo, los ritmos de adaptación no eran todo lo adecuado que se requería. Es por esto cómo los diferentes atentados que se producen a partir de septiem-

9 Doc.19/1, de 23 de enero de 1999.

10 Punto 43.1. a) del Plan de Acción de Viena.

11 Poco después, a través de la Recomendación del Consejo de 9 de diciembre de 1999 sobre la cooperación en materia de lucha contra la financiación del terrorismo se recomienda a los servicios de seguridad nacionales que intercambien regularmente la información de que dispongan sobre las estructuras y métodos empleados en la financiación de grupos terroristas que operen en más de un Estados miembros.

12 Documento del Consejo 10010/3/04 REV 3. En mayo de 2005 se establece el nuevo Plan de Acción que recoge la Estrategia de la UE para combatir el terrorismo. 
bre de 2001 marcan con claridad los estadios que vivirá la cooperación en materia de inteligencia.

La sensación de vulnerabilidad que aquel día compartieron ciudadanos y gobernantes en todo el mundo llevó a la rápida adopción de medidas y compromisos políticos para afrontar una amenaza terrorista vivida en directo. El presidente de la Comisión Europea Romano Prodi se aprestó al día siguiente de los atentados a mostrar su solidaridad con los Estados Unidos. Esta solidaridad se reafirmó el 14 de septiembre en una declaración conjunta de los Jefes de Estado o de Gobierno que, con el fin de contribuir a la instauración de un marco internacional de seguridad y prosperidad, se comprometieron a que "la PESD debía hacerse operativa tan pronto como fuera posible y para ello se hará un esfuerzo para fortalecer la lucha contra el terrorismo". Por otra parte, instaron a todos los países a intensificar sus esfuerzos en el combate contra el terrorismo y anunciaron el compromiso de la Unión en favor del surgimiento de un movimiento antiterrorista fuerte y duradero a escala mundial.

El 19 de septiembre la Comisión presentó dos propuestas de decisión marco del Consejo: una sobre la lucha contra el terrorismo, que tiene por objeto aproximar las legislaciones de los Estados miembros en materia de delitos y penas en este ámbito, y otra sobre la Orden de Detención Europea y los procedimientos de entrega de personas entre Estados miembros, que prevé un nuevo sistema que sustituya a los mecanismos actuales de extradición basado en el principio de reconocimiento automático o casi automático de las decisiones judiciales de detención tomadas en otro Estado miembro.

El 20 de septiembre se celebró una reunión ministerial Unión Europea-Estados Unidos, en la que participaron el ministro de Asuntos Exteriores de Bélgica y Presidente en ejercicio del Consejo, Patten, miembro de la Comisión, Solana, Alto Representante de la PESC, y Powell, Secretario de Estado americano. En una declaración común, los participantes destacaron la importancia de una acción conjunta para combatir el terrorismo y afirmaron su resolución de actuar en el ámbito de la seguridad aérea y de los otros medios de transporte y en el de la cooperación policial y judicial, sobre todo en materia de extradición, de prohibición de financiación del terrorismo, de control en las fronteras y de intercambio de datos electrónicos.

Estas reuniones e iniciativas culminaron en la reacción más contundente y concreta a los atentados contra los Estados Unidos. El 21 de septiembre, y a propuesta de Alemania, se celebra un Consejo Europeo Extraordinario para reafirmar la solidaridad de la UE hacia los Estados Unidos y durante el cual se aprueba el Plan de Acción contra el terrorismo. ${ }^{13}$ La presidencia de turno belga agrupó todas las tareas en un Plan de Acción de la Unión con más de 70 medidas individuales que desde entonces está siendo constantemente actualizada. Además de los proyectos judiciales y de política interior,

13 http://www.europarl.eu.int/summits/pdf/bru_fr.pdf 
estas medidas individuales incluyen tareas de política exterior, transporte y financieras relativas a la guerra contra el terrorismo como, por ejemplo, la estabilización de la situación en Afganistán.

De este Consejo Europeo surge el compromiso de que "los Estados miembros compartan con Europol, sistemáticamente y sin retraso, toda la información útil relativa al terrorismo". ${ }^{14}$ Por su parte, un día antes, y también reunidos en sesión extraordinaria, los ministros de Justicia e Interior habían unas conclusiones relativas a la mejora de la cooperación judicial, policial y entre los servicios de información y seguridad del Estado, la lucha contra la financiación del terrorismo así como medidas de protección de las fronteras enfatizando la necesidad de la cooperación entre los servicios de inteligencia de los Estados miembros. ${ }^{15}$

Se decidió establecer dentro de Europol, y por un período inicial de seis meses, un Grupo de Trabajo Contraterrorista al cual los Estados miembros están invitados a "designar a oficiales de enlace de la policía y los servicios de inteligencia especializados en la lucha contra el terrorismo". ${ }^{16}$ Esto último era un importante logro ya que el primer ministro belga Guy Verhofstadt se lamentó tras los atentados de que durante los dos años anteriores había intentado establecer una política antiterrorista empleando Europol sin alcanzar resultado alguno. ${ }^{17}$ Sería por decisión del Consejo de 21 de septiembre de 2001 cuando se invita a los Estados a nombrar a un oficial de enlace de cuerpos policiales y agencias de inteligencia especializados en la lucha contra el terrorismo sin prejuicio de sus respectivas legislaciones con la tarea de recopilar toda la información e inteligencia relevante concerniente a las amenazas actuales como analizar la información colectiva y llevar a cabo análisis estratégicos y operativos. ${ }^{18}$

El posterior Consejo de Laeken, en diciembre de 2001, tendría necesariamente como condicionante el 11-S, además de la revisión de todo lo que la Unión había hecho previamente en materia de seguridad y lucha contra el crimen. A partir de este Consejo se llegan a diversos acuerdos políticos que fraguan en la adopción de diferentes instrumentos judiciales, muchos de los cuales ya estaban concebidos desde $1987 .{ }^{19}$ La primera de ellas es la Orden Europea de Detención que eliminaba las órdenes de extradición habituales. ${ }^{20}$ La segunda medida fue una Decisión Marco que proporciona

14 Conclusiones y Plan de Acción para el Consejo Europeo Extraordinario, de 21 de septiembre de 2001, http://ue.eu. int/eu/Info/eurocouncil/index.html, parágrafo 3.

15 Consejo de Justicia e Interior de 20 de septiembre de 2001, doc.SN 3926/6/01 REV 6. Véase sobre todo el apartado "Cooperation between police and intelligence services".

16 Consejo de Justicia e Interior, doc.SN 3926/6/01 REV 6, 20 de septiembre de 2001.

17 http://www.eupolitix.com

18 Conclusiones y Plan de Acción para el Consejo Europeo Extraordinario, de 21 de septiembre de 2001, http://ue.eu. int/eu/Info/eurocouncil/index.html, parágrafo 10.

19 Por ejemplo, pocos meses antes se había adoptado una Decisión Marco del Consejo de 26 de junio de 2001 sobre blanqueo de dinero, identificación, rastreo, congelación, incautación y confiscación de instrumentos procedentes del crimen, JO L 182, 5 de julio de 2001. Véase también http://ue.eu.int/ejn/indexfr.htm

20 Decisión Marco del Consejo, 2002/584/JAl, de 13 de junio relativa a la Orden de Arresto Europeo y procedimientos de entrega entre Estados miembros. JO L 190 de 18 de julio de 2002. 
una definición común de terrorismo y unas penas mínimas debido a que sólo seis Estados miembros tenían legislaciones específicas sobre terrorismo. ${ }^{21}$ No obstante, la voluntad política no siempre estaba acorde con ciertos problemas que surgieron a la hora de establecer esta definición.

La tercera de ellas se recoge en la Decisión Marco del Consejo de 13 de junio de 2002, sobre la lucha del terrorismo. Establece que los Estados miembros adoptarán las medidas necesarias para que se consideren delitos de terrorismo los actos intencionados tipificados como delitos según los respectivos Derechos nacionales. ${ }^{22}$ Finalmente, se establecen grupos especiales de trabajo e investigación. ${ }^{23}$ La presidencia de turno griega fue encargada de continuar con la aplicación del Plan de Acción de la UE para el combate del terrorismo y el fortalecimiento de la cooperación policial y judicial en el campo del combate contra el terrorismo y se llega al compromiso de intensificar la cooperación e intercambio de información entre servicios de inteligencia y la frecuencia de las reuniones regulares de los jefes de los servicios de inteligencia nacionales. ${ }^{24}$

Otra de las medidas que brotan al albur de los atentados es la Decisión 2003/48/JAI del Consejo de 19 de diciembre de 2002, relativa a la aplicación de medidas específicas de cooperación policial y judicial en la lucha contra el terrorismo y que marca el inicio de una importante etapa que tendrá su continuación con la iniciativa sueca de mejorar el intercambio de información e inteligencia. Sin embargo, la conciencia de la necesidad de incrementar este intercambio ha llevado a que el Consejo adopte la Decisión 2005/671/ JAl, de 20 de septiembre de 2005, relativa al intercambio de información y a la cooperación sobre delitos de terrorismo que indicaba con claridad que los objetivos sólo pueden conseguirse mediante la cooperación entre los Estados miembros y, por consiguiente, debido a la necesaria reciprocidad, pueden lograrse mejoras a nivel de la Unión, ésta puede adoptar medidas de acuerdo con el principio de subsidiariedad.

Su artículo 2 indica que cada Estado miembro designará entre sus servicios policiales un servicio especializado $u$ otras autoridades responsables del cumplimiento de la ley, que, de conformidad con la legislación nacional, tendrá acceso a toda la información pertinente relativa a las investigaciones criminales por delitos de terrorismo, recabará esa información y la remitirá a Europol dentro de lo contenido en el artículo 2.6, que establece que "cada Estado miembro tomará las medidas necesarias para garantizar que toda información pertinente contenida en cualquier documento, expediente, dato, objeto u otro medio de prueba, intervenido o confiscado durante investiga-

21 Para mayor profundidad véase Foreign Office, Counter-Terrorism Legislation and Practice: A Survey of Selected Countries, octubre de 2005. www.fco.gov.uk/Files/kfile/QS\%20Draft\%2010\%20FINAL1.pdf

22 Decisión Marco 2002/475/JAI de 13 de junio de 2002, DO L 164, 22 de junio de 2002, p. 3.

23 Decisión Marco 2002/465/JAl, de 13 de junio; Relativa a los equipos de investigación conjuntos, JO L 162 de 20 de junio de 2002.

24 Consejo Europeo. Conclusiones de la Presidencia, Tesalónica 19 y 20 de junio de 2003. Anexo I. 
ciones o procesos penales seguidos en relación con delitos de terrorismo pueda estar disponible lo antes posible". Se comprueba con rotundidad cómo el Consejo Europeo insta a los Estados miembros a que capaciten a Europol para que, en cooperación con Eurojust, desempeñe un papel clave en la lucha contra las formas graves de delincuencia transfronteriza (organizada y no organizada) y de terrorismo. Pero en otros ámbitos fuera del policial, la cooperación también se ha incrementado. Así, en el Consejo Europeo de Sevilla en junio de 2002 se adoptó una declaración sobre la contribución de la PESC, incluida la PESD, a la lucha contra el terrorismo, que ha permitido avances sustantivos en cuestiones como la cooperación en materia de inteligencia en el contexto de la cooperación con la OTAN. ${ }^{25}$ Por su parte, la Asamblea de la UEO manifestó en diciembre de 2003 que en lo respecta a "la búsqueda y diseminación de inteligencia, el primer paso debe ser hacer un mejor uso de las capacidades nacionales existentes, ya que el problema no es meramente de falta de capacidades". ${ }^{26}$ A partir de aquí nace una larga carrera por poner orden en los identificadores biométricos y bases de datos que tuvo un largo recorrido, sobre todo por las presiones del gobierno norteamericano que fijó finales de 2003 como fecha límite. ${ }^{27}$

\section{Atentados de Madrid}

La propia ciudad de Madrid albergó el 14 de marzo de 2004 una cumbre de servicios de inteligencia del G5, esto es, Alemania, Francia, Reino Unido, Italia y España donde acuerdan establecer una red de intercambio de información sobre el terrorismo internacional y un sistema de alerta rápida entre los países sobre el robo de explosivos, armas o sustancias nucleares, radiológicas, biológicas o químicas susceptibles de ser utilizadas en atentados terroristas. ${ }^{28}$

Precisamente tres días antes del 11-M, Javier Solana había finalizado un informe interno en el que se identificaban tres grandes carencias en los esfuerzos antiterroristas: algunos Estados miembros no había implementado los acuerdos de la Unión tales como la Orden Europea de Detención, había carencia de recursos para jugar un papel significativo en la lucha contra el terrorismo y la coordinación entre los funcionarios de la Unión trabajando en campos policiales, asuntos exteriores y políticas de defensa era muy pobre. No es así extraño que tras los atentados, la Comisión Europea, en su reunión de 18 de marzo de 2004, confeccionara un Plan de Acción en respuesta a los atentados terroristas en Madrid, cuyo objetivo es realzar

25 Declaración del Consejo Europeo sobre la contribución de la PESC, incluida la PESD, en la lucha contra el terrorismo. Anexo V de las Conclusiones de la Presidencia, SN200/02, Sevilla, 21 y 22 de junio de 2002.

26 Declaración del Consejo Europeo sobre la contribución de la PESC.

27 Propuesta de la Comisión sobre identificadores biométricos para visa y permiso de residencia para nacionales de terceros países, doc.IP/03/1289, 24 de septiembre de 2003.

28 Finalmente, y tras intensísimas y reiteradas peticiones, Polonia se ha incorporado a este grupo en la reunión de ministros del Interior en Helligendamm (Alemania) los días 22 y 23 de marzo de 2006. 
la coordinación operativa y la cooperación y que formula con claridad los puntos clave que la Unión considera para el tema de la inteligencia. ${ }^{29}$

En este Plan de Acción se manifiesta que "no podemos continuar produciendo redes e instituciones y rechazar dotarlas con los instrumentos necesarios para llevar a acabo sus trabajos o simplemente o usándolos. Mucho debe ser hecho". ${ }^{30}$ En primer lugar, "la Unión debe establecer un nuevo mecanismo de coordinación para el intercambio de información donde las fuerzas policiales, autoridades judiciales y servicios de inteligencia realcen la confianza mutua y el intercambio operativo de inteligencia, permitiendo que realicen su trabajo adecuadamente. Europol y Eurojust debe implicarse plenamente, junto con las inteligencias nacionales y las redes judiciales como ya prevé el Tratado. Las prioridades de intercambio de información de esos mecanismos deben ser establecidos por el Consejo, aunque las actividades prioritarias serían: método de reclutamiento de las organizaciones terroristas; identificación de las células durmientes, sus fuentes de financiación en la sociedad civil y sus relaciones exteriores."

El documento continúa afirmando que "el intercambio de información entre las autoridades nacionales y el nivel de la UE debe incrementarse dramáticamente. Una cierta cultura de secreto es comprensible pero sólo a primera vista ya que ha probado ser contraproducente. Más que nuevas instituciones u organismos, la Unión tiene que avanzar con una visión comprensiva sobre la metodología para el intercambio de información. El nuevo mecanismo de coordinación no puede ser una CIA europea sino sólo un instrumento del segundo pilar. El terrorismo es el principal problema, sobre todo a nivel interior, y por lo tanto los mecanismos que sugerimos se establezcan deberán intercambiar información principalmente en el tercer pilar. En definitiva, se apela por poner en coordinación las diferentes instituciones antes que consumir tiempo y recursos destruyendo y creando nuevas organizaciones.

En segundo lugar, "la Unión debe trabajar hacia la implementación de una política europea de información con objetivos de aplicación de la ley. Existen fuerzas policiales con un importante componente de actividad de inteligencia y efectivos sistemas nacionales de inteligencia criminal que son compatibles al nivel de la UE y facilitan el acceso efectivo, análisis y uso de datos debe ser desarrollado. Tal política de información debe permitir facilitar la detección de amenazas al orden público y la seguridad, evitar riesgos a la seguridad y luchar contra el crimen organizado y el terrorismo a través de la Unión incluyendo favorecer el acceso a datos no producidos con fines de aplicación de la ley. En tercer lugar, la Comisión está preparada para explorar la creación de sinergias entre los existentes y futuros sistemas

29 European Commission action paper in response to the terrorist attacks on Madrid. MEMO/04/66, Bruselas 18 de marzo de 2004.

30 European Commission action paper in response to the terrorist attacks on Madrid. MEMO/04/66, Bruselas 18 de marzo de 2004. 
de información (SIS II, VIS y Eurodac) con vistas a explotar su valor añadido en la lucha contra el terrorismo en el marco de sus respectivos marcos legales y técnicos.

En lo relativo al aspecto organizativo se establece que Europol - que ya tiene un mandato antiterrorista - deberá ser usado más y mejor. Los Estados miembros necesitan proporcionarle la información e inteligencia que necesita para realizar su trabajo, en particular en el área de la prevención e investigación del ataque terrorista, y no olvidemos que el 15 de marzo el Consejo Europeo había decidido establecer en el seno de Europol un Grupo de Trabajo Terrorista. ${ }^{31}$ En quinto lugar, el Grupo de Trabajo de Jefes de Policía debe tener un papel más fuerte en las actividades operativas concernientes a la prevención y lucha contra el terrorismo. Eurojust debe tener un papel más destacado en la lucha contra el terrorismo y el Consejo debe darle un mandato más claro para coordinar las actividades de las autoridades fiscales nacionales a través de la Unión en lo relativo al terrorismo.

Por su parte, en el Consejo informal de ministros de Defensa de Zaragoza, de 22 y 23 de marzo de 2004, el ministro español presentó el conjunto de iniciativas impulsadas por la Presidencia española con el objetivo de inscribir la lucha contra el terrorismo dentro de la política de defensa. Entre ellas destacaba la necesidad de impulsar la cooperación de los servicios de inteligencia militar. ${ }^{32}$ Por su parte, "en la crucial área de la inteligencia, el reciente establecimiento de una unidad de análisis dentro de la Secretaría del Consejo representa un paso concreto hacia una coordinación más efectiva entre los Estados miembros al respecto."

A pesar de la voluntad de mejora que nace tras el 11-S, la Declaración del Consejo Europeo sobre el combate al terrorismo de 25 de marzo de 2004 entona un muy necesario mea culpa sobre las cosas que aún no se han hecho. ${ }^{33}$ En esta Declaración se da instrucciones al Consejo para que "estudie medidas legislativas con el fin de simplificar el intercambio de información e inteligencia entre las autoridades encargadas de garantizar le cumplimiento de la ley en los Estados miembros" debido a "la compartimentación de la información, así como la falta de una política clara de canales de información obstaculizan ese libre intercambio" y habla de la existencia de problemas "legales, técnicos y prácticos que dificultan el intercambio entre

31 Secretaría del Consejo, doc.7906/04 JAl 100, de 25 de marzo de 2004.

32 http://ue.eu.int/ueDocs/cms_Data/docs/pressdata/ES/discours/69917.pdf

33 De hecho se inicia una intensa actividad normativa para mejorar las carencias detectadas. En pocos meses la Comisión publica un total de cinco Comunicaciones desde el 11 de marzo de 2004: "Sobre la mejora del acceso a la información por parte de las autoridades encargadas de garantizar el cumplimiento de la ley", COM 429, de 16 de junio de 2004; "Prevención, preparación y respuesta a los ataques terroristas", COM 698 de 20 de octubre de 2004; "Prevención y lucha contra la financiación del terrorismo", COM 700 de 20 de octubre de 2004; "Lucha contra el terrorismo: preparación y gestión de las consecuencias", COM 701 de 20 de octubre de 2004 y "Protección de las infraestructuras críticas en la lucha contra el terrorismo", COM 702 de 20 de octubre de 2004. 
los Estados miembros multiplican las dificultades para superar la división de la información entre los diversos ministerios nacionales". ${ }^{34}$

Finalmente, el Consejo Europeo acordó en marzo de 2004 que el Coreper tuviera un papel mucho más importante en la coordinación de los pilares en la lucha contraterrorista, algo en lo que la información ocupa un destacado papel. Se establece también la figura del coordinador contraterrorista cuya misión será "trabajar en el marco de la Secretaría del Consejo, y coordinará el trabajo del Consejo en combatir el terrorismo y, con el debido respeto a las responsabilidades de la comisión, mantener una panorámica de todos los instrumentos disponibles de la Unión con vistas a informar regularmente al Consejo y el seguimiento de sus decisiones"; 35 una figura que había sido propuesta en el Consejo de Lisboa durante la presidencia irlandesa.

En junio de 2004, la Comisión habla de la posibilidad de establecer una Política Europea en Inteligencia e Información con la posibilidad de proveer mejor información sobre canales seguros y establecer una infraestructura a nivel europeo para este intercambio consiguiendo un modelo de cooperación en inteligencia criminal que permita "la sincronización de los asesoramientos sobre una metodología común, sistemático escrutinio de las amenazas por sectores de vulnerabilidad y los recursos humanos y materiales necesarios". ${ }^{36}$ Un buen instrumento para ello sería la designación de Puntos Nacionales Centrales de Contacto (PNCC) para el intercambio internacional de información a los que, unos meses después, el Programa de La Haya dedicaría cierta atención.

Toda esta toma de conciencia sobre lo deficiente de la cooperación en información e inteligencia fraguaron en la propuesta de Decisión Marco del Consejo sobre la simplificación del intercambio de información e inteligencia entre los cuerpos de seguridad de los Estados miembros de la Unión Europea, en particular en relación con delitos graves, incluidos los actos de terrorismo de 4 de junio de 2004. ${ }^{37}$ En ella se establece lo perentorio del "intercambio de información e inteligencia sobre la delincuencia y las actividades delictivas es la base de la cooperación policial en la Unión para alcanzar el objetivo general de mejorar la seguridad de sus ciudadanos". Para alcanzarlo se hace fundamental "el acceso rápido a una información e inteligencia fiables y actualizada para que los cuerpos de seguridad puedan prevenir, localizar e investigar con éxito delitos y actividades delictivas,

34 Nota del Coreper al Consejo. Informe al Consejo Europeo sobre la implementación de la Declaración sobre el combate del terrorismo, doc.10009/3/04, de 11 de junio de 2004.

35 Consejo de la Unión. Nota de prensa "El Consejo Europeo se centra en la lucha contra el terrorismo", PRES 04/81/2004.

36 Comunicación de la Comisión al Consejo y al Parlamento Europeo sobre la mejora del acceso a la información por parte de las autoridades encargadas de garantizar el cumplimiento de la ley (Política de información de la UE). COM 429 final, de 16 de junio de 2004

37 Propuesta de Decisión Marco del Consejo sobre la simplificación del intercambio de información e inteligencia entre los cuerpos de seguridad de los Estados miembros de la Unión Europea, en particular en relación con delitos graves, incluidos los actos de terrorismo, doc.10215/04, de 4 de junio de 2004. A efectos de su gestación véase la Comunicación de la Comisión al Consejo y al Parlamento Europeo "Hacia el fortalecimiento del acceso a la información por parte de las fuerzas policiales", doc.10745/04 ENFOPOL 77, de 22 de junio de 2004. 
en particular en una zona en la que se han suprimido los controles en las fronteras interiores. Puesto que el terrorismo y otras actividades delictivas graves se cometen de forma clandestina, es necesario controlarlas, e intercambiar con especial rapidez la información sobre los sospechosos".

Un punto destacado en todas estas normas es el del principio de acceso equivalente a los datos de las autoridades encargadas de garantizar el cumplimiento de la ley. El primer objetivo fundamental de la política de información para garantizar el cumplimiento de la ley es la libre circulación de la información entre los servicios competentes, incluidos Europol y Eurojust. Actualmente, las autoridades competentes pueden utilizar bases de datos accesibles a nivel nacional. Sin embargo, las autoridades de otros Estados miembros no tienen acceso en la práctica, debido a la cantidad de problemas que se les plantean. La política de información pretende hacer accesible esta información a todas las autoridades de la UE, incluidos Europol y Eurojust, con el fin de asistirlas en el cumplimiento de sus funciones y de conformidad con el Estado de Derecho.

Técnicamente, en lo que respecta al intercambio y tratamiento de datos, además del principio de acceso equivalente, otra forma de mejorar el acceso a datos y bases es incluyéndolos en una red o creando bases de datos centrales. En este contexto, el Consejo Europeo invitó a la Comisión "a presentar propuestas para mejorar la compatibilidad entre bases de datos europeas (SIS-II, VIS y Eurodac) con el fin de aprovechar su valor añadido dentro de los respectivos marcos legales y técnicos de prevención y lucha contra el terrorismo". ${ }^{38}$ Para alcanzar estos objetivos debería concebirse un enfoque en etapas basado en la creación de formatos armonizados de codificación de los datos y de normas de acceso a los diversos sistemas. En las primeras rondas de consultas de la Comisión Europea con las partes implicadas se detectaron problemas que pueden dificultar el intercambio de información. ${ }^{39}$

Las medidas y técnicas encaminadas a crear un clima de confianza son de importancia fundamental (normas y metodologías comunes). Pero además es fundamental en la política de información proponer las medidas necesarias para desarrollar un sistema represivo basado en la inteligencia a escala de la UE que sería un Modelo Europeo de Inteligencia. Otro objetivo sería contribuir a la instauración de un clima de confianza entre las autoridades represivas, los funcionarios y demás partes interesadas europeas estableciendo una plataforma común de valores, normas y orientaciones políticas compartidas.

El Consejo Europeo de 5 de noviembre de 2004 aprobó un nuevo programa para el desarrollo de un área de Libertad, Seguridad y Justicia en

38 Secretaría del Consejo, doc.7906/04 JAl 100, de 25 de marzo de 2004.

39 Declaración de Dublín y Conclusiones de las reuniones del Foro europeo sobre prevención de la delincuencia organizada. 
la Unión conocido como el Programa de la Haya que establece la agenda en el campo de la Justicia e Interior para los próximos cinco años. ${ }^{40} \mathrm{El}$ programa de La Haya es el sucesor del programa de Tampere, ${ }^{41}$ y en él se identifica la renuencia a compartir información como uno de los mayores problemas, especialmente grave en temas como el contraterrorismo, lo que se achacaba a la falta de confianza, y se recomienda la interoperabilidad de las bases de datos y la designación de PNCC. ${ }^{42}$

Es clave entender que a estas alturas se ve cómo caminar hacia un modelo de inteligencia criminal europeo es una de las tareas más importantes por delante en este campo, y que requerirá de los esfuerzos compartidos de los Estados miembros y los organismos e instituciones de la Unión. Este intercambio de información debe ir más allá del meramente estratégico para abordar la amenaza del crimen organizado de cuya lucha indica que "requiere mejorar la cooperación entre los cuerpos de la ley (policía, justicia, aduanas) así como hacer completo uso del potencial desarrollo de Europol y Eurojust. Porque aunque al terrorismo internacional, tras el 11-S se le ha otorgado un papel especial dentro de los nuevos riesgos y amenazas a la seguridad, ${ }^{43}$ no podemos olvidar otras amenazas.

El propio programa de La Haya reconoce que "el refuerzo de la cooperación policial requiere la atención concentrada en la confianza mutua y en su consolidación". Pero la Unión no sólo lo deja en el intercambio de información sino que también "deberá velarse por la coordinación de las actividades operativas de los cuerpos y fuerzas de seguridad y otros organismos en todos los espacios de libertad, seguridad y justicia y el Plan de La Haya se basa en el principio de "disponibilidad de la información" para todas las agencias policiales a lo largo de la Unión. ${ }^{44}$ En su punto segundo (consolidación de la seguridad) este programa se dedica a la mejora del intercambio de información y sostiene que el Consejo entiende que es necesario un planteamiento innovador del intercambio transfronterizo de información policial. Por este motivo se establece que a partir del 1 de enero de 2008, el intercambio de información deberá regirse respecto al principio de disponibilidad, lo que significa que, en todo el territorio de la Unión, un funcionario de policía de un Estado miembro que necesite información para llevar a cabo sus obligaciones pueda obtenerla de otro Estado miembro y que el organismo policial del otro Estado miembro que posea dicha información la facilitará para el propósito indicado, teniendo en cuenta el requisito de las investigaciones en curso en dicho Estado.

40 Consejo Europeo. Conclusiones de la Presidencia, 4 y 5 de noviembre de 2004, doc.14292/04, p. 27-28. http:// ue.eu.int/uedocs/cmsUpload/EU_4.5-11.pdf

41 A Response to The Hague Programme: Protecting the Rule of Law and Fundamental Rights in the Next Five Years of an EU Area of Freedom, Security and Justice?. http://www.libertysecurity.org

42 Sobre estos PNCC véase el informe del Parlamento británico "HM Customs and Excise and the European Police Office"; EUROPOL, PAC 97-98/434. Report by the Comptroller and Auditor General.

43 Véase el informe "2002 EU Organised Crime Report", Europol 2530-108 rev1, 3 de octubre de 2002.

44 Consejo Europeo. Conclusiones de la Presidencia, 4 y 5 de noviembre de 2004, doc.14292/04, p. 27-28. http:// ue.eu.int/uedocs/cmsUpload/EU_4.5-11.pdf 
Se plantea también mejorar la Decisión 2003/48/JAl del Consejo de 19 de diciembre de 2002 relativa a la aplicación de medidas específicas de cooperación policial y judicial en la lucha contra el terrorismo, ampliando el ámbito de aplicación de los intercambios de información sobre todas las infracciones en materia de terrorismo, siendo Europol y Eurojust los destinatarios de esta información. ${ }^{45}$ Esta Decisión había sido adoptada sobre la base de una iniciativa de España para contribuir a mejorar los intercambios de información sobre las investigaciones y a los procesos penales. Indica que Europol y Eurojust deberían ser destinatarios de esta información ya se trate de información sobre las investigaciones, procesos o condenas por delitos de terrorismo. En sus conclusiones habla de la "necesidad de la mejora cualitativa y cuantitativa de información", y propone un enfoque por etapas sucesivas: "en la lucha contra el terrorismo es esencial que los servicios pertinentes puedan disponer de la información más completa y actualizada, según sus ámbitos de competencia, condenas incluidas."

Tras los atentados de Madrid se fue muy crítico con la incapacidad de Europol para liderar el proceso de intercambio de información porque es demasiado rígido y hay reticencia de los servicios de inteligencia a aceptarlo como socio. Uno de los problemas clave es la reticencia de los Estados miembros a transmitir datos de los servicios de información e inteligencia a Europol, lo que afecta a su capacidad para suministrar el valor añadido necesario a los análisis de la delincuencia a nivel de la Unión Europea. En consecuencia, los Estados miembros consideran con frecuencia que Europol no satisface las demandas de sus clientes -las fuerzas del orden- con lo que no están motivados a suministrarles más información. ${ }^{46}$

El 15 de marzo de 2005, el G5 decidió en una cumbre informal en Granada crear una base de datos conjunta de los sospechosos de actividades terroristas y establecer un único punto de contacto por el robo o pérdida de explosivos, armas o sustancias nucleares, radiológicas, biológicas o químicas susceptibles de ser utilizadas en atentados terroristas. El 15 de abril de 2005 se reunieron Europol y el Sitcen para informarse de sus planes sobre el combate terrorista. Pero poco después tiene lugar un acuerdo entre diferentes Estados miembros muy significativo a nuestros efectos. El 27 de mayo de 2005, Bélgica, Alemania, España, Francia, Luxemburgo, Holanda y Austria firman en Prüm (Alemania) un Tratado relativo a la profundización de la cooperación transfronteriza, en particular en materia de lucha contra el terrorismo, la delincuencia transfronteriza y la migración ilegal. Entre otras cosas, este Tratado introduce medidas para la mejora del intercambio de información sobre ADN y huellas dactilares. El Tratado está abierto a la

45 Comunicación de la Comisión al Consejo y al Parlamento Europeo relativa a determinadas acciones que deben emprenderse en el ámbito de la lucha contra el terrorismo y otras formas graves de delincuencia, en particular, con el fin de mejorar los intercambios de información. COM 221 final, de 29 de marzo de 2004.

46 Documento de la Comisión sobre el terrorismo a la atención del Consejo y destinado a alimentar los debates del Consejo Europeo, doc. SEC 348, de 18 de marzo de 2004. 
adhesión de cualquier Estado miembro de la Unión Europea y las partes contratantes tienen como objetivo incorporar las disposiciones del Tratado al marco jurídico de la Unión Europea. ${ }^{47}$

Pocas semanas después, en la reunión de ministros de Justicia e Interior en La Haya, los responsables de estas áreas analizaron medidas para que a partir del 1 de julio de 2005, el Sitcen y Europol, en colaboración con los Estados miembros pasasen a preparar regularmente una evaluación de la amenaza terrorista en la Unión Europea y recomendar medidas apropiadas. ${ }^{48}$ Plantean también que, a partir del 1 de enero de 2006, la estrategia de la Unión Europea sobre radicalismo y reclutamiento de terroristas, aúne aspectos como integración, educación, vigilancia y seguridad así como reforzar los mecanismos de relación entre Europol y Eurojust. En esta reunión también se plantearon que, a partir del 1 de enero de 2008, el intercambio de información en temas de libertad, seguridad y justicia fuese ya total entre los Veinticinco. Desgraciadamente, pocas jornadas después, el terrorismo golpearía de nuevo el corazón de una ciudad europea.

\section{Atentados de Londres}

Tras los atentados en el metro de Londres del mes de julio de 2005, la Unión relanza su compromiso en la lucha contra el terrorismo. Charles Clarke, el ministro del Interior británico - país sobre el cual precisamente recaía la presidencia de turno de la Unión - convoca para el día 13 un Consejo Extraordinario de Justicia e Interior en el cual se declara como inmediata prioridad el construir dentro del marco de la Unión, un espacio de colaboración, para perseguir e investigar el terrorismo más allá de las fronteras nacionales. ${ }^{49}$ Además, el Consejo urgió a los Estados miembros a "compartir información, garantizar la coordinación y permitir toma de decisiones colectivas en emergencias, particularmente por ataques terroristas en más de un Estado miembro". ${ }^{50}$

Antonio Vitorino defendía que "escaseando el compartir información, la verdadera llave maestra de la lucha conjunta contra el terrorismo" ningún sistema de seguridad europeo efectivo es posible. La Comisión sugirió la máxima expansión del intercambio de información sobre terrorismo y especialmente el desarrollo de bases de datos de personas y organizaciones sospechosas de terrorismo y desarrollo de un dossier europeo de personas condenadas para finales de 2004..$^{51}$ La Unión está trabajando en otras medidas como son la inclusión de pasaportes biométricos para fortalecer

\footnotetext{
47 Secretaría del Consejo, "Tratado de Prüm", doc.10900/05, de 27 de mayo de 2005.

48 Para esta evaluación véase la Comunicación de la Comisión al Consejo y al Parlamento Europeo "Evaluación de las políticas de la UE en materia de libertad, seguridad y justicia", COM 332 final, de 28 de junio de 2006.

49 Comunicado de prensa tras el Consejo Extraordinario de Justicia e Interior, 13 de Julio de 2005. http://ue.eu.int/ ueDocs/cms Data/docs/pressData/en/jha/85703.pdf

50 Comunicado de prensa tras el Consejo Extraordinario de Justicia e Interior, 13 de Julio de 2005

51 Declaraciones del Comisario de Justicia e Interior. IP/04/425.
} 
el control de las fronteras, la adhesión de los Estados al Plan de Acción y especialmente a la Orden Europea de Detención y a los equipos de investigación conjunto, facilitar la formación conjunta de miembros de policía y equipos de emergencia y finalmente, fortaleciendo las capacidades de la Unión para combatir el financiamiento y lavado de dinero.

El 21 de septiembre de 2005, en una Recomendación del Parlamento Europeo al Consejo Europeo y al Consejo sobre el intercambio de información y cooperación relativo a amenazas terrorista, se establece que dada la importancia de los datos de tráfico para la prevención, investigación, detección y enjuiciamiento de delitos graves, como el terrorismo y la delincuencia organizada, según demuestran la investigación y la experiencia práctica de varios Estados miembros, existe la necesidad de asegurar que los datos tratados por proveedores de comunicaciones electrónicas al ofrecer servicios públicos de comunicaciones electrónicas o redes públicas de comunicación se conservan durante un determinado periodo de tiempo. ${ }^{52}$

Sólo dos meses después se producen los primeros resultados. La Decisión del Consejo 2005/671/JAl de 20 de septiembre de 2005 establece que los Estados miembros transmitirán información a Europol y Eurojust referente a investigaciones, procesamientos y condenas penales correspondientes a delitos de terrorismo. ${ }^{53}$ Esta decisión también indica que los Estados miembros -pero no se refiere específicamente a los servicios de seguridad e inteligencia- también desempeñan un cometido esencial en la prevención del terrorismo en el marco de sus actividades de recogida y producción de inteligencia para preservar la seguridad nacional.

La consideración tercera de esta Decisión del Consejo afirma que "es esencial, en la lucha contra el terrorismo, que todos los servicios implicados puedan disponer de la información más completa y actualizada, según sus ámbitos de competencia. A raíz de esta consciencia la Decisión del Consejo establece un mecanismo para garantizar la transmisión a Europol de la información resultante de las actividades de los servicios de seguridad e inteligencia de los Estados miembros, relativas a delitos de terrorismo. También establece un mecanismo para garantizar el intercambio de información sobre delitos de terrorismo entre los servicios de seguridad e inteligencia de los Estados miembro. En el mes de noviembre se establece que cada Estado miembro deberá designar a uno o más autoridades centrales para recibir y enviar esta información. En caso de una condena en un Estado miembro, la autoridad nacional debe informar a la contraparte de cuyo Estado es nacional el condenado.

Pero los esfuerzos no han terminado con este conjunto normativo. En las conclusiones de la presidencia austríaca de la Unión se establecía la

\footnotetext{
52 Recomendación del Parlamento Europeo al Consejo Europeo y al Consejo sobre el intercambio de información y cooperación relativo a amenazas terrorista COM 438 final, de 21 de septiembre de 2005.

53 DO L 253, de 29 de septiembre de 2005, p.22.
} 
necesidad de nuevos esfuerzos, en particular, en lo referente $a^{54}$ la continuación de los avances realizados en el Sistema de Información de Schengen (SIS II) y en la aplicación del acervo de Schengen en los nuevos Estados miembros. ${ }^{55}$

La presidencia finlandesa de la Unión, iniciada el 1 de julio de 2006, vuelve a dejar claro que para luchar contra el terrorismo y prevenir la radicalización y el reclutamiento es necesario un mejor intercambio de información entre las fuerzas policiales y las autoridades judiciales. Reaparece una vez más el principio de disponibilidad para iluminar esta relación de la que dice debe convertirse en la piedra angular del intercambio de información para principios de 2008. También prestará atención a la protección de datos que este intercambio puede generar antes de aplicar este principio y buscará impulsar la cooperación operativa a nivel de la Unión y en cuya base está una buena relación entre Europol y Eurojust. ${ }^{56}$

La Presidencia checa de la Unión, inaugurada el 1 de enero de 2009, incluye en su agenda importantes temas de seguridad y cooperación, como ya se había indicado en el avance de las presidencias francesa, checa y sueca. ${ }^{57}$ En primer lugar, se busca promover el desarrollo de Schengen, sobre todo en la segunda generación, conocida como Schengen II así como la implementación del VIS. El terrorismo también se ha convertido por naturaleza propia en un objetivo de la presidencia con la implementación del Action Plan on Enhancing the Security of Explosives, pero el principal aspecto será el trabajo sobre el nuevo plan que sustituya al Programa de La Haya, que finaliza en 2009. Será, así necesario, adoptar uno nuevo plan que dé respuesta a los retos y objetivos para el campo de Justicia e Interior que refleje la nueva situación en la UE tras la ampliación a 27 Estados miembros para lo cual trabajará el Future Group. ${ }^{58}$

En el campo de la cooperación judicial en temas criminales, la presidencia checa quiere proponer una nueva Decisión marco que creará un mecanismo para prevenir y resolver conflictos de competencia en los procedimientos criminales entre Estados miembros que se basará en el intercambio de información en procedimientos judiciales en curso, mecanismo que ya venía siendo reclamado desde 2007, ante las carencias que mostraba la Decisión marco de intercambio de información e inteligencia.

54 Consejo Europeo. Conclusiones de la Presidencia, doc.10633/1/06 REV 1 CONCL 2, de 15 y 16 de junio de 2006

55 No olvidemos que el intercambio de información entre policías era uno de los principios del pacto de preacceso para los nuevos países. Acuerdo de preacceso, principios 3 y 4.

56 Agenda preliminar para la presidencia finlandesa de la Unión Europea del segundo semestre de 2006 http://www.eu2006.fi/the_presidency/en_GB/presidency_agenda/_files/75429538661335079/default/preliminary_ agenda.pdf.

57 http://www.eu2009.cz/en/czech-presidency/programme-and-priorities/programme-and-priorities-479.

58 Este grupo fue establecido por sugerencia del ministro del Interior alemán Wolfgang Schäuble durante la presidencia alemana en la reunión informal de ministros del Interior en Desden, y cuya primera reunión tuvo lugar en Eltville, Hesse, el 20 y 21 de mayo de 2007. El grupo está presidido por el vicepresidente Frattini y los seis ministros del interior de la actual y próxima troika (Alemania, Portugal y Eslovenia, así como Francia, República checa y Suecia), un representantes de la subsiguiente presidencia: España, Bélgica o Hungría, y expertos de los diferentes Estados miembros según se considere. 
En el proceloso proceso de aprobación del Tratado de Lisboa hubo cambios, aunque pocos afectaron a lo que el texto recogía para el área de Libertad, Seguridad y Justicia. Se establece un mayor protagonismo de los Parlamentos nacionales en la supervisión de Europol y la evaluación de las actividades de Eurojust conforme al artículo 86 de la nueva versión. Por otra parte, el establecimiento de una Fiscalía Europea a partir de Eurojust, que ya había sido previsto en el Artículo III-274 del Tratado Constitucional y ha sido mantenida en Lisboa. El establecimiento de una Fiscalía Europea responsable de la investigación y apertura de juicio sobre crímenes considerados como graves y de naturaleza transfronteriza conllevaría su transformación en un nuevo y poderoso actor comunitario en el área de cooperación judicial en la UE, no obstante, a tenor del propio artículo 83, sería necesaria unanimidad en el Consejo previa aprobación del Parlamento Europeo.

Otro de los elementos que permanecen del anterior Tratado es la creación en el seno del Consejo de un Comité Permanente en materia de Seguridad Interior del nuevo artículo 71 TFUE. Su objetivo sería garantizar una cooperación operacional más decisiva en materia de seguridad interior de la Unión. Además, tendría como objetivo favorecer la cooperación y coordinación de la actuación de las autoridades competentes de los Estados miembros. Sin embargo, su composición y competencias siguen siendo difusas. Esta cooperación se fortalece en el ámbito policial, en cuyo artículo 88 TFUE establece que "la Unión desarrollará una cooperación policial en la que participen todas las autoridades competentes de los Estados miembros, incluidos los servicios de policía, los servicios de aduanas y otros servicios con funciones coercitivas especializados en la prevención y en la detección e investigación de infracciones penales", y a estos efectos, el Parlamento Europeo y el Consejo podrán adoptar, con arreglo al procedimiento legislativo ordinario, medidas relativas a "la recogida, almacenamiento, tratamiento, análisis e intercambio de información pertinente", con lo que la fluidez con la que el impulso político a la cooperación se incrementa notablemente.

El Programa de Estocolmo es el mayor éxito en materia de Seguridad, Libertad y Justicia de la presidencia sueca - heredado por la presidencia española -, y que deberá desarrollar el programa plurianual 2010-2014.59 En él se plantea la necesidad de revisar los instrumentos actualmente existentes y crear un modelo europeo para el intercambio de información. Esta estrategia de gestión de la información tendría como ejes: i) un desarrollo operativo (intercambio de información y de sus instrumentos regido por las necesidades policiales), y ii) la interoperatividad de los sistemas informáticos, velando por una total conformidad con los principios de la protección de datos y la seguridad de los datos al desarrollar dichos sistemas. En este esquema, Europol debería ser el eje para el intercambio de información

59 Programa de Estocolmo. http://elsjpresidencia.files.wordpress.com/2010/01/programa-de-estocolmo-aprobadopor-el-consejo-europeo-de-diciembre-de-2009.pdf 
entre las autoridades policiales de los Estados miembros, para la creación de un prestador de servicios y una plataforma para los servicios policiales.

La presidencia española de 2010 no planteó grandes avances, pero probablemente tampoco deberíamos esperar grandes innovaciones cuando nos encontramos con un área que empieza a estar consolidada y cuando Estocolmo ha marcado claramente su agenda. ${ }^{60}$ Así, la Presidencia española lanza una Estrategia de Seguridad Interior (ESI) que analiza riesgos, las amenazas y vulnerabilidades, y define prioridades estratégicas. La definición de la ESI es una iniciativa española que pretende unificar en un solo texto todas las medidas que se han ido adoptando a lo largo de los años en esta materia y dotarlas de "un paraguas que marque las líneas estratégicas marco". ${ }^{61}$ Esta estrategia subraya la prevención y la anticipación, que se basa tanto en un enfoque proactivo y dirigido hacia la inteligencia, como a la obtención de pruebas para el proceso judicial. Enfatiza, así, que sólo es posible llevar a cabo una acción legal con éxito si se dispone de toda la información necesaria.

Por su parte, el Comité de Seguridad Interior (COSI) fue creado el 25 de febrero de 2010, siguiendo las directrices marcadas por el Tratado de Lisboa. Su objetivo es intensificar la coordinación de las actuaciones operativas entre los Estados miembros de la UE en materia de seguridad interior. EI COSI tiene, en primer lugar, la misión de evaluar la cooperación operativa en la UE -identificará las deficiencias y recomendará medidas oportunas para corregirlas-. En caso de ataques terroristas o grandes catástrofes, el Comité ofrecerá asistencia al Consejo de Justicia e Interior. Otra de las tareas del COSI será la coordinación de la acción de las agencias europeas con competencias en materias de justicia e interior, como Europol, Eurojust, FRONTEX, y CEPOL, a las que reconoce el importante y creciente papel desarrollado, y para el cuál requieren de un intenso intercambio de información.

Plantea el desarrollo de un modelo integral basado en el intercambio de información y la confianza mutua, que culmine en el principio de disponibilidad de la información. Para que las autoridades en materia de seguridad puedan prevenir y actuar anticipadamente deben disponer, en el momento adecuado, del mayor número de datos posibles sobre hechos delictivos y sus autores, modus operandi, tipología de las víctimas, vehículos utilizados etc. En esta línea busca la creación de un Modelo Europeo de Intercambio de Información seguro y estructurado que incluiría todas las bases de datos de la UE que resulten relevantes para asegurar la seguridad en la UE, permitiendo la interacción entre éstas. La presidencia española enfatiza algo que ha quedado claro en los últimos años: máximo intercambio de información e inteligencia, pero con pleno respeto al derecho a la privaci-

60 Programa de la Presidencia Española de la Presidencia del Consejo. http://www.eu2010.es/export/sites/presidencia/ comun/descargas/programa_ES.PDF

61 Estrategia de Seguridad Interior de la Unión Europea. Hacia un modelo europeo de seguridad Febrero 2010. http:// tsa.ondemand.flumotion.com/tsa/ondemand/PEUE/PDF/Seguridad_ES.pdf 
dad y la protección de datos personales, aspectos todos ellos en los que coinciden las posteriores presidencias belga, húngara y polaca, conforme se recoge en sus agendas.

\section{Conclusiones}

1- La cooperación en materia de inteligencia siempre ha existido entre los países de la Unión, sobre todo en amenazas, más que en potencialidades. Los diferentes acuerdos existentes, entre ellos el más relevante de Trevi, han supuesto la base para una cooperación que, necesariamente, se ve incrementada con los atentados del 11 de septiembre contra los Estados Unidos y que supone un punto de inflexión que se refuerza tras los atentados de Madrid.

2 - Las manifestaciones de los actores demuestran con claridad cómo antes que ir hacia fórmulas complejas de coordinación, la Unión apuesta por puntos intermedios. En éste, los Estados miembros y sus respectivas estructuras de seguridad e inteligencia, y también judiciales, tendrán un papel muy relevante. Será sobre ellas sobre quienes recaiga el componente operativo, que la Unión parece no estar ni en deseo ni en capacidad de absorber.

3 - La creación de una Política Europea de Inteligencia se está fraguando poco a poco. Pero no como una política omini compresiva para todas las áreas de trabajo de la UE sino para algunas muy determinadas. Si bien comenzó como soporte técnico para la PESC, la amenaza terrorista ha provocado que ésta se fundamente más sólidamente en el área de Justicia e Interior. Así Europol y Eurojust se busca comenzar a caminar en la amenaza criminal quedando la inteligencia de tipo estratégico reservada a los ámbitos de la PESC y de la gestión de crisis. El papel que le queda a la Unión es el de coordinación.

4 - Lograr esta política requiere de un fluido intercambio de información. La desconfianza internacional puede ser comprendida pero no la interna entre agencias nacionales. Los suecos propusieron que el acceso fuera en condiciones no más estrictas que para las agencias nacionales, algo que no sería posible si no hubiera igualdad de formatos y de traducciones. No obstante, hay que avanzar en esta idea del intercambio multilateral y el principio de acceso equivalente.

5 - La creación de una Agencia Europea de Inteligencia que venía siendo reclamada desde hace más de una década ha sido descartada. Reactivada la petición con los atentados de Madrid, el posicionamiento de los diferentes actores ha sido muy claro. Los grandes países de la Unión confían en sus capacidades, por lo que no pretenden crear una gran agencia que en esencia duplicaría sus capacidades nacionales y que serviría para dar apoyo a los países pequeños. Estos, por su parte, reclaman este tipo 
de agencia, al ser muy conscientes de su incapacidad para garantizarse su seguridad en un escenario tan complejo. Finalmente, las instituciones comunitarias han optados por seguir profundizando en la cooperación y en los instrumentos existentes, que en crear nuevos órganos y capas en la estructura de la Unión. 